Original Article

\title{
Occupational justice health questionnaire: reflections on its application ${ }^{1}$
}

\section{Questionário de saúde e justiça ocupacional: reflexôes em sua aplicação}

Michael Palapal Sy, (D), Ma. Patricia Nicole Ragunton Roraldo c (D), Rod Charlie Razon Delos Reyes (D), Daryl Patrick Gamboa Yao ${ }^{\mathrm{b}}$ (D), Roi Charles Sarmiento Pineda ${ }^{\mathrm{d}}$ (D)

${ }^{a}$ University of the Philippines Manila - UPM, Manila, Philippines.

${ }^{\text {b}}$ Tokyo Metropolitan University - TMU, Tokyo, Japan.

${ }^{\mathrm{c}}$ Mariveles Mental Hospital, Bataan, Philippines.

${ }^{\mathrm{d}}$ Katholieke Universiteit Leuven - KUL, Leuven, Belgium.

How to cite: Sy, M. P., Roraldo, M. P. N. R., Delos Reyes, R. C. R., Yao, D. P. G., \& Pineda, R. C. S. (2021). Occupational justice health questionnaire: reflectons on its application. Cadernos Brasileiros de Terapia Ocupacional, 29, e2961. https://doi.org/10.1590/2526-8910.ctoAO2244

\begin{abstract}
Introduction: Occupational justice concept is defined as the equal access and participation in all occupations, including the resources required for participation and opportunities for social inclusion. Occupational justice not only remains conceptually ambiguous for occupational therapy practice but also lacks critical examination of its applicability to non-Western cultures. Objective: This article describes the reflexive process exercised by the researchers during and after using the Occupational Justice Health Questionnaire (OJHQ) as an occupational therapy tool in a substance addiction rehabilitation setting in the Philippines. Method: This qualitative study gathered transcriptions from field notes and focus group discussions of the authors. These transcriptions were characterized by the authors' reflections on their experiences using the OJHQ on four distinct periods: 1) OJHQ translation, 2) pilot testing, 3) OJHQ-Filipino version administration, and 4) project conclusion. Data were analyzed through thematic analysis. Results: Qualitative data revealed four themes: tool usefulness; administration guidelines; contextualized use of the tool; and nuances of occupational injustices in substance addiction and rehabilitation setting. Conclusion: Findings expanded OJHQ's purpose to enhance clinical reasoning, promote justice-oriented health care, activate reflective practice, and recognize the interweaving and evolving nature of injustices. This article offers an understanding of how reflections can facilitate cultural adaptation of practice tools used in occupational therapy and justice practice.
\end{abstract}

Keywords: Qualitative Analysis, Cultural Competence, Social Justice, Occupational Therapy.

\footnotetext{
${ }^{1}$ Ethical Approval: This study received ethical approval from the Department of Science and Technology-Central Luzon Health Research and Development Consortium Protocol No. 2018-10 Protocol No. 2018-10 and the Tokyo Metropolitan University Arakawa Campus-Research Safety Ethics Committee Protocol No. 18011Protocol No. 18011.
} 


\section{$\underline{\text { RESUMO }}$}

Introduçáo: $\mathrm{O}$ conceito de justiça ocupacional é definido como a igualdade de acesso e participação em todas as ocupaçôes, incluindo os recursos necessários para a participaçáo e oportunidades de inclusão social. A justiça ocupacional não apenas permanece conceitualmente ambígua para a prática, mas também carece de um exame crítico de sua aplicabilidade a culturas não ocidentais. Objetivo: Descrever o processo reflexivo dos pesquisadores durante e após o uso do Questionário de Saúde e Justiça Ocupacional (OJHQ), como uma ferramenta em terapia ocupacional em um ambiente de reabilitação para pessoas com dependência de substâncias psicoativas nas Filipinas. Método: Estudo qualitativo baseado em notas reflexivas e grupos focais. As transcriçóes foram caracterizadas pelas reflexóes dos autores sobre suas experiências usando o OJHQ em quatro períodos distintos: 1) Tradução do OJHQ, 2) teste piloto, 3) Administração da versão OJHQ-Filipino e 4) conclusão do projeto. Os dados foram analisados por meio de análise temática. Resultados: Os dados qualitativos revelaram quatro temas: utilidade da ferramenta; diretrizes de administraçáo; uso contextualizado da ferramenta; e nuances de injustiças ocupacionais no contexto de dependência de substâncias psicoativas e reabilitação. Conclusão: Houve a expansão do propósito do OJHQ para aprimorar o raciocínio clínico, promover cuidados em saúde orientados para a justiça, ativar a prática reflexiva e reconhecer a natureza entrelaçada e evolutiva das injustiças. Compreendese como as reflexóes podem facilitar a adaptaçáo cultural das ferramentas práticas utilizadas na terapia ocupacional com base em práticas de justiça.

Palavras-chave: Análises Qualitativas, Competência Cultural, Justiça Social, Terapia Ocupacional.

\section{Introduction}

Occupational justice is considered as an outcome of intervention (American Occupational Therapy Association, 2020) and aspect of contexts and environments within the occupational therapy's domain of practice (Bailliard et al., 2020). The occupational justice concept is defined as the "[...] access to and participation in the full range of meaningful and enriching occupations afforded to others, including opportunities for social inclusion and the resources to participate in occupations to satisfy personal, health, and societal needs" (American Occupational Therapy Association, 2020, p. 79). Although the utility of occupational justice in different contexts and cultures remains ambiguous for some occupational therapists (Durocher et al., 2014; Gupta, 2016; Hammell, 2017), a scoping review by Malfitano et al. (2019) ascertained that occupational justice and related concepts are already informing OT practice through diverse approaches. While occupational justice remains to be conceptually redefined, occupational scientists and justice workers take advantage of opportunities to enact and promote justice not only at the level of individuals, but for groups, communities, and populations (Bailliard, 2016; Malfitano et al., 2016).

The Occupational Justice Health Questionnaire (OJHQ) (Wilcock \& Townsend, 2014) was developed to aid occupational therapists in identifying specific injustices experienced by individuals, groups, or communities. The OJHQ is a five-part checklist 
with binary options (i.e., able and unable) across 27 items related to basic needs (Part I), overall wellbeing (Part II), living standards (Parts III \& IV), and specific injustices decreasing occupational participation (Part V). The OJHQ has been used for crosssectional and case studies in the Philippines particularly in the context of substance addiction and rehabilitation (Sy et al., 2019; Sy \& Ohshima, 2019) and students' experiences during the coronavirus pandemic (Delos Reyes et al., 2021). The main role of the OJHQ was to aid in identifying determinants that characterize a person or group's ability to engage in a variety of daily occupations, ability to exercise basic human rights within a certain context, and inability to participate due to existing occupational injustices.

However, there is a need to critically reflect on their use of the OJHQ for at least two reasons. First, there is a lack of definition for items and guidelines in administering the OJHQ. On one hand, this allows for flexible use in diverse practice settings. On the other hand, there is a risk for inconsistency in responses due to differing definitions used, particularly when administered by different assessors. The lack of definitions and abstractness of terms in the OJHQ may also pose as a challenge for translations to another language (de Groot, 1992). Thus, establishing definitions of terms and constructs can help occupational therapists explain the nature of their practice better (Kennedy \& Davis, 2017) i.e., explicating the nature of occupational injustices to inform clientele evaluation, intervention, and discharge plans. Second, occupational justice, as with many concepts in OT, has been developed with a "Western perspective" in mind (Nilsson \& Townsend, 2010, p. 58). Several researchers (Hammell \& Iwama, 2012; Malfitano et al., 2019) have criticized this aspect of occupational justice and called for more cultural diversity in OT and occupational justice research.

Since the 1970s, Filipino researchers have highlighted the problem pertaining to the unsuitability of foreign-developed tests and the paucity of locally made tests (GuanzonLapeña et al., 1998; Magno, 2010). This is true not only in occupational therapy (Coronel, 2005) but also in other professions like psychology (Bernardo, 2011) and speech-language pathology (Bondoc et al., 2017), just to name a few. The indiscriminate adaptation and use of "imported" assessments like the OJHQ may fail to measure their intended constructs if cultural aspects are not considered (Coronel, 2005). Several factors that favor the use of foreign-made tests over locally developed ones include the multitude of languages in the Philippines, bilingualism and English proficiency of the majority of Filipinos, and xenocentric tendency of Filipinos (i.e., more favorable attitudes towards Western ideas and products; Bernardo, 2011; Enriquez, 1992; See, 2008). However, it has been criticized that such practice promote the colonizing Anglo-American bias and dominance in professional discourses (See, 2008).

Reflecting on occupational justice-inspired tools of practice, such as the use of OJHQ, in non-Western cultures like the Philippines could facilitate more culturally nuanced ways of evaluating and theorizing occupational justice. Jasso (2005) asserts that while some elements of justice are universal, culture may strongly influence group- or individualspecific conceptions of justice. What is considered just and fair and how individuals respond to perceived injustices can vary appreciably between groups and populations (Leung \& Stephan, 2001). For example, the Philippines is a country with a predominantly conservative Catholic population. Catholic philosophy views suffering, i.e., experiencing injustice, as penance for one's sins, an essential part of humanity, and/or a way to be closer to God (Garcia, 2006). Thus, individuals may, from their own perspective, consider 
experiences of injustice as something that they deserve and/or must wholeheartedly welcome. Additionally, rampant corruption and violence of law enforcement has instilled normalcy of these occurrence in the minds of Filipinos, which was reinforced further by the country's brutal War on Drugs (Jensen \& Hapal, 2018). Jensen et al. (2013) conducted a victimization survey to measure the level of violence in a local community, specifically to assess the people's perception about violence, the nature of these violent acts, and what do people do to seek redress. Findings from this survey revealed that Filipinos describe the experience of police violence and corruption as ok lang which from the Tagalog language means "just okay" because it is expected and not a cause of complaint (Jensen et al., 2013). These examples show that some experiences (e.g., lack of access to healthcare or leisure pursuit) may not be readily labelled by Filipinos as occupational injustices considering that extortion perpetuated by the police is ok lang (Jensen \& Hapal, 2018).

The reflections produced and analyzed for this study were based on the researchers' work on translating and administering the OJHQ (Sy et al., 2019), which can provide groundwork to support its use in the Philippine context. It is, however, important to note that the data in this article were simultaneously collected with the larger study of Sy et al. (2019), but the data analyses were done separately hence producing findings related to the reflections of those who used the OJHQ as a tool rather than the experiences of those who were interviewed using the tool. Reflexive exercises support the recognition of personal biases and predispositions and normalizes the consideration of multiple perspectives within and outside therapy (Bailliard et al., 2020). In consideration of the rich and diverse culture of the world and unique justice norms in each culture, this current study aims to: 1 ) describe the reflexive process exercised by the researchers during and after the conclusion of the first author's doctoral project regarding occupational justice as applied in the substance addiction rehabilitation setting in the Philippines; and, 2) promote critical reflexivity in adopting the OJHQ through the detailed account of the researchers' experiences.

\section{Methods}

This qualitative study is based on the reflections of reflective researchers who used the OJHQ in a substance addiction rehabilitation center in the Philippines. The reflective process was supported by the use of reflective notes furnished across four reflective periods: OJHQ translation (Step 1), pilot testing (Step 2), administration (Step 3), and project conclusion (Step 4). The authors of the OJHQ expressed their consent in using the instrument to gather data about the experiences of the tool's utility within the Filipino population (E. Townsend, personal communication, 26 November 2017). This study was reviewed and approved by the institutional ethics board in Japan and in the Philippines.

\section{Reflective researchers}

The authors of this paper served as the reflective researchers. The first three authors (referred to as MS, NR, and CDR, respectively) actually experienced using the OJHQ-Filipino Version (FV; see Supplemental Document 1), which was translated using the four-stage translation proposed by the World Health Organization (2020). The translation involved forward translation, backward translation, and tool revisions. The reflective researchers were 
Philippine-licensed occupational therapists who participated in the translation and administration of the OJHQ-FV in substance addiction rehabilitation facilities in the Philippines. None of the reflective researchers were employed or affiliated in the research sites.

At the time of the research, MS (32, male) was a full-time doctoral student in OT at a Japanese university with a 10-year experience in clinical practice, teaching, and research in OT. CDR (26, male), a holder of master's degree in OT, was an OT instructor at a Philippine university and concurrently working in a pediatric clinic; and NR (25, female) was a master's student in OT employed in a mental health facility. In terms of experience in qualitative research, MS completed a qualitative research (phenomenology) as an output for his doctoral dissertation, while CDR and NR had some experience in qualitative research but had considerable experience in interviewing. To harmonize tool use and note taking between the reflective researchers, MS conducted training sessions with CDR and NR. DGY and RCP are master and doctoral students, respectively, and had experience in designing, implementing and writing qualitative research.

Qualitative researchers have been increasingly integrating their positionality statement within their research to provide an overall orienting lens especially in studies that address gender, class, race, and injustice issues in society (Creswell, 2014). In this study, the researchers posit that without intentional investigation and cultural adaptation, occupational therapy tools that are underpinned by Western constructs will render assessment results and/or outcome measurements that could further marginalize individuals.

\section{Data collection procedures}

Data were gathered throughout the four reflective periods (from Step 1 to Step 4) through field notes, focus group discussions, meeting notes and exchanges via email and instant messaging. All data were written electronically and by hand. The field notes guide proposed by Phillippi \& Lauderdale (2018), which are summarized in Table 1, were used flexibly for Steps 1 to 3 in order to uncover and document contextual factors and nuances through the use of field notes. Doing so would ensure that the researchers co-created field notes that are structured, detailed, and in-depth, which are consistent with qualitative data analytic approaches (Phillippi \& Lauderdale, 2018).

In Step 1, reflections were drawn from the reflective researchers' personal judgments, note exchanges, and declared biases during the translation processes. In Step 2, reflections were largely extracted from the pilot testing session on three residents confined in one of the substance addiction rehabilitation center. In Step 3, reflections were based on the experience in the OJHQ-FV administration to 24 residents from two substance addiction rehabilitation centers. In Step 4, the reflections were drawn from the insights from the data analysis period until the project's conclusion. Reflective researchers had a focus group discussion at the conclusion of each step. The first three authors invited both DGY and RCP to join during Step 4 not only to help in writing the paper but to also critically reflect on the results and discuss the concept of occupational justice based on the reflective notes collected and processed. Figure 1 illustrates the steps of OJHQ use during the doctoral project and reflection moments, as well as guide the questions during each respective reflection moment.

An integral part of documentation and analysis in qualitative research, field notes were the primary data collected, organized, and analyzed to address the current research 
objective. For this study, these field notes contained the reflexive data produced by the reflective researchers. Observational data drawn largely from field notes can fill gaps of information that are missed or misunderstood when conducting interviews because of their openness (Phillippi \& Lauderdale, 2018). The three reflective researchers were oriented at the very beginning of the translation process that the data that was being collected for the phenomenological study (Sy et al., 2019) was separate from the reflection data being gathered, but the collection of these data sets were to be done simultaneously. One hour after every interview session, field notes were independently produced and were then discussed and shared among MS, NR, and CDR for feedback and debriefing. At the conclusion of Step 3 (which lasted four days), a two-hour debriefing session was done to confirm accuracy and completeness of field notes before conducting data analysis. To facilitate on-going reflections beyond the debriefing sessions, the authors also allowed for the collection of e-mail correspondence, group chat messages, and videoconference meeting notes to be considered in the analyses.

\section{Data analysis}

The data analysis followed an inductive thematic analysis approach which included open coding, creating categories, and abstraction (Elo \& Kyngäs, 2008). Data from the field notes were transferred and collated in a word document in preparation for the open coding phase assisted by the ATLAS.ti 7 software (Friese, 2013). The first thematic analysis was carried out on data collected during the first three reflection moments (i.e., OJHQ translation, pilot testing, and OJHQ-FV administration). A second analysis was done at the conclusion of the doctoral project (i.e., last reflection moment).

For Steps 1 to 3, MS implemented the open coding which involved reading and rereading all data and subsequently creating a "code" that describes the related quotations from the field notes. Quotations linked to a code formed the basis of each code's operational definition. CDR and NR reviewed all codes and their respective operational definitions for clarity, accuracy, and conciseness. Categories and themes were then created individually by MS, NR, and CDR while keeping in mind that categorization must be done openly, generate tentative explanations, and increase the likelihood of addressing the research aim. The reflective researchers used the cutting and sorting techniques (Ryan \& Bernard, 2003) manually and digitally for the creating categories phase. After independently creating categories and themes, the reflective researchers convened to formulate the themes. During Step 4, the formulated themes were critically examined by all authors to ensure reflexivity and to counter-check if the themes answered the research aims.

\section{Rigor and trustworthiness}

It is also important to take note that while this study partially involved a translation of a tool, which traditionally warrants positivist measures such as validity and reliability, a qualitative inquiry such as in the case of this study requires measurements of value and effectiveness via trustworthiness constructs (Collier-Reed et al., 2009). Rigor and trustworthiness were employed using the strategies outlined by Guba \& Lincoln (1989). In particular, credibility, dependability, and conformability measures were considered. 
STEP
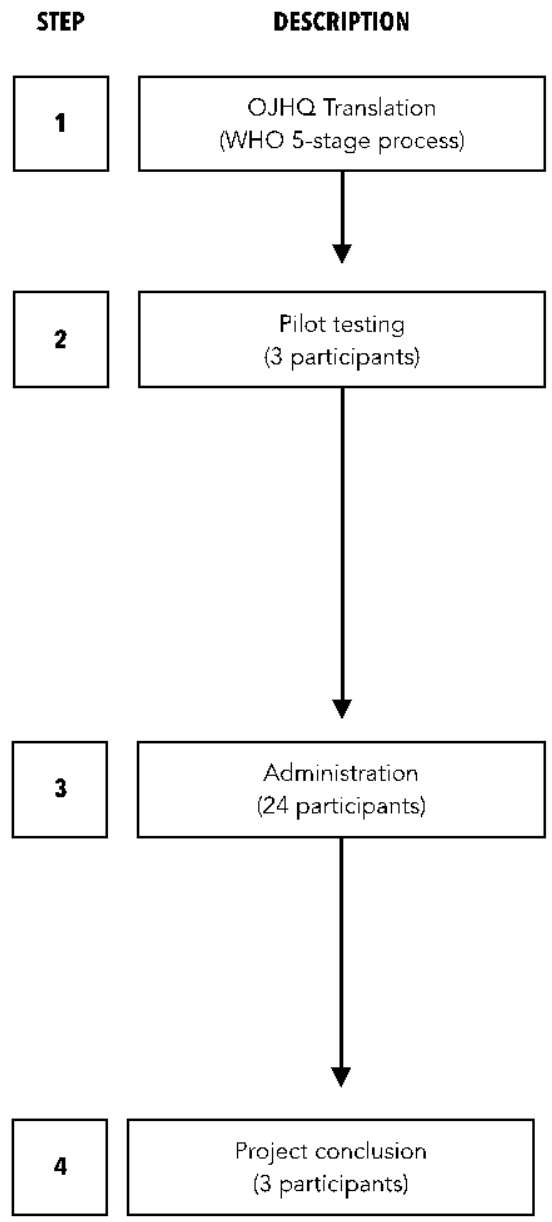

\section{REFLECTION}

\section{Reflections on the translation} and pilot testing

1. What do you think is the question asking?

2. What came to your mind when you hear the term/phrases?

3. Explain why you did not understand the term?

4. Is there a better alternative term to facilitate understandability?

5. What were the challenges?

6. What are your personal biases?

7. What are your personal judgments?

Reflections on the administration

1. What are the challenges experienced while administering the OJHO-FV?

2. What are the advantages of administering the OJHO-FV

3. What are the other observations and comments that you have during the administration experience?

Reflections on the construct "occupational justice"

1. How was the concept of occupational justice understood by the residents?

2. How does the unique characteristics/ demographics of the residents shape their experience of occupational (in)justice?

Figure 1. The reflection process on the translation, administration, and experience of using the OJHQ-FV.

Table 1. Selected field notes components considered during the focus group discussion (Phillippi \& Lauderdale, 2018).

\begin{tabular}{ll}
\hline - Atmosphere & $\bullet$ Overall thoughts (strengths and limitations) \\
\hline - Non-verbal behaviors & $\bullet$ Potential biases \\
\hline - Over-all depth of content & $\bullet$ Reflection on interview facilitation \\
\hline - Interaction among participants & $\bullet$ Thoughts on the interview \\
\hline - Depth of response & $\bullet$ Changes for the future \\
\hline - Value of question & $\bullet$ Saturation \\
\hline
\end{tabular}


Credibility was enhanced since the reflective researchers had several engagements with the research site including correspondence with authorities, ocular visits, discussing methodological procedures with the people from the research sites, and scheduled peer debriefing. To further increase the credibility of this study, MS conducted an orientation and demonstration sessions with NR and CDR on how the interview should be administered using the OJHQ. This procedure is important to ensure that the reflective researchers are not just professionally qualified to administer an assessment tool but have the capacity to employ the interview with practical skills (interviewing), theoretical knowledge (occupational justice and occupational rights), and critical thinking (clinical reasoning). Moreover, the reflective researchers followed the WHO four-stage translation process and sought assistance for official forward translation from English to Filipino (Sentro ng Wikang Filipino [Center of Filipino Language], University of the Philippines Manila) and backward translation from Filipino to English (Lexicode, Inc.). All translators were bilingual speakers who are proficient in both English and Filipino. The pilot testing allowed the researchers to fine tune the translation. Dependability was also established because of the adherence to a detailed study protocol and documentation of decision trail via an inquiry audit. This means that in every partial result from data collection and analysis, a consultation among the first three authors occurred before proceeding to the next step (reflexivity). Data were transcribed, encoded, and analyzed considering varied points-of-view in interpreting the study findings through regular meetings and collaborative writing. Moreover, the manuscript was subjected to external feedback by MS's Ph.D. supervisor and two colleagues (one is an experienced OT educator, while the other is a seasoned researcher) external to the study. The last two authors (RCP and DGY) of this article-who did not administer the OJHQ-FV-were invited to co-construct the manuscript towards its final stages (i.e., from finalization of themes until submission for publication) to employ investigators triangulation. While an operational data saturation (i.e., quantify the number of new codes per interview over time) was not done, theoretical data saturation was attained through regular meetings where an iterative process of thematization was employed until no new information was discovered to inform the emergent themes. Lastly, confirmability was established since the reflective researchers gathered field notes separately and were then discussed collectively during scheduled debriefings and meetings. During these meetings, reflexivity was employed to ascertain that preconceived biases were recognized and that different perspectives were considered.

\section{Results}

From our analyses of the reflections on translating and administering the OJHQFV, four themes emerged: 1) usefulness of the OJHQ-FV, 2) guidelines in OJHQ-FV administration, 3) contextualization of the OJHQ-FV, and 4). The nuances of occupational injustices in the substance addiction rehabilitation setting (see Table 2). 
Table 2. Themes that emerged from reflective researchers' field notes.

\begin{tabular}{l}
\hline Theme 1: Usefulness of the OJHQ \\
\hline Sub-theme 1.1: Enables narrative clinical reasoning \\
\hline Facilitates story-telling \\
Emancipatory approach \\
\hline Facilitates advocacy/self-advocacy \\
\hline Sub-theme 1.2: Extracts additional layer of non-clinical data \\
Sensitivity, variability, and novelty of data gathered \\
Practicality in translating abstract concepts to concrete issues \\
Identification of specific social conditions \\
\hline Theme 2: Guidelines in OJHQ administration \\
\hline Sub-theme 2.1: User's competencies \\
\hline Demonstrates adequate preparation for and administration of OJHQ \\
\hline Requires advanced interviewing skills and training \\
\hline Facilitates a relaxed atmosphere \\
Acknowledges personal bias \\
\hline Sub-theme 2.2: Situational considerations \\
\hline Observations on physical space, socio-cultural context, and gestures \\
\hline Tools needs to be used at least twice \\
\hline Sub-theme 3.1: Tool-related issues \\
\hline Related and repeating items \\
Inapplicability of some items because of its Western underpinnings \\
Inapplicability of some items \\
\hline Sub-theme 3.2: Further refinement processes \\
Population specific \\
\hline More comprehensive definition of items needed \\
Translation and interpretation considerations \\
Instrument format and layout \\
\hline Ignorance of (in)justice is a reality \\
\hline The interweaving and evolving nature of occupational injustice \\
\hline Theme 4: The nuances occupational injustices in the substance addiction rehabilitation setting \\
\hline Contextualization of the OJHQ \\
\hline
\end{tabular}

\section{Theme 1: Usefulness of the OJHQ}

The first theme revealed an expanded purpose of OJHQ in OT practice. Two subthemes emerged: enabling narrative clinical reasoning (sub-theme 1.1) and extracting additional layers of non-clinical data (sub-theme 1.2).

All reflective researchers found that enabling narrative clinical reasoning - the act of making sense of a person's life story as it impacts daily living (Boyt Schell, 2014) — when using the OJHQ required the facilitation of story-telling, an emancipatory approach, and a clear understanding of the conceptual underpinnings of occupational justice. MS stated that "[...] the mode of interviewing is like story-telling... which requires the interviewer to interpret stories and conversations". While the OJHQ was not intended to be used as an intervention strategy for this study, MS observed that several residents in the substance addiction rehabilitation center described the interview experience as 
positive and cathartic and disclosed that being able to share their stories was important because it gave an assurance that someone cared enough to listen. Here is a sample quotation from one of the interviewees that characterized catharsis:

People with money do not like what is happening because they are protected by their own money... They can't have the guts to get angry at criminals who are on drugs because rich people do not understand what we experience under the poverty line! (Sy et al., 2019, p. 9).

MS, NR, and CDR unanimously agreed that the openness felt by some residents during the sessions stemmed from free sharing of information about pertinent topics including personal matters, family concerns, politics, injustices, human rights violations, personal abilities, and motivations. Bringing the residents' narrative into consciousness and enhancing awareness of individual rights could potentially lead to more proactivity towards self-advocacy. This was supported by MS's comment, "OJHQ-FV was able to capture personal, sensitive, and critical client narratives that can be used for advocacy". CDR also acknowledged the potential use of OJHQ-FV for advocacy, which constitutes actions that promote justice and empower individuals to seek and obtain resources to support health, well-being, and occupational participation (American Occupational Therapy Association, 2020).

Extracting additional layers of non-clinical data also emerged as another purpose of the OJHQ-FV. Although the tool contained items pertaining to a person's health producing clinically relevant data (i.e., recovery status [demographic data], social, physical, and mental well-being [Part II]; United Nations Rights_-living standard adequate for health and wellbeing [Part III]), these data sets also largely described the residents' health in relation to occupational justice and occupational rights, hence producing non-clinical data. These nonclinical data sets pertained to constructs characterized by social determinants of health and were perceived by the reflective researchers as sensitive, variable, novel, and helpful in informing practice. Although similar occupational injustice experiences were identified across accounts, these were interpreted from different perspectives with respect to the residents' diverse background in terms of education, origins, and upbringing. NR reflected that "...the tool captured a completely new perspective on human experiences that is not usually extracted from regular [OT] interviewing, history-taking, or quantitative instruments".

Through the OJHQ-FV, "abstract concepts were identified and concretized in the form of goals and strategies for intervention" according to MS. For instance, poverty was identified to be an injustice. The multiple aspects of poverty was examined by reviewing and threading the stories given by the residents, which allowed for the identification of specific social conditions underlying occupational injustice like relational concerns (family and friends), restrictive or loose labor policies, living environments (physical and social), and cultural mindsets. While addressing injustices could be cumbersome or out of the scope of practice of an occupational therapist, obtaining such knowledge drawn from these non-clinical data sets were perceived to aid in formulating concrete action plans and recommendations at the last part of the OJHQ.

\section{Theme 2: Guidelines in the OJHQ administration}

The second theme was characterized by information on how to administer the OJHQ-FV based on the OJHQ-FV users' experience and reflections. This theme had 
two subthemes: users' competencies (sub-theme 2.1) and situational considerations (sub-theme 2.2).

The users' competencies are skills that OJHQ users must possess. An essential competency was demonstrating adequate preparation prior to administration including arranging logistics, reviewing medical records, and inspecting the interview space. Competencies in advanced interviewing must also be in place including clarifying the purpose of the tool to the resident (i.e., research versus care planning) and utilizing an interview guide with flexibility. The administration of the interview was crucial. For instance, addressing each other by first names made the residents feel more dignified and the interviewers more approachable. The use of non-verbal communications including silence likewise supported an atmosphere of respect for the residents and genuine interest in the residents' personal stories. MS mentioned that leaning slightly forward and gesturing an open posture can be helpful, especially because most residents were withdrawn. NR also observed that strategies for active listening, (e.g., nodding to demonstrate acknowledgement) helped facilitate the conversation. Apart from ensuring that the physical space was relaxed, ensuring safe and comfortable researcher-resident interaction was also a recognized competency. MS wrote, “...one way of facilitating a relaxed atmosphere is [...] by facilitating storytelling to identify injustice experiences and by assuring the residents that they are not being checked, monitored, or diagnosed".

Acknowledging personal bias when conducting research is a fundamental competency. NR remarked that she had a tendency to listen more to their stories which made it challenging for her to terminate some interviews that went beyond the 90minute mark. CDR also pointed out during debriefing that MS had a former family who had drug addiction problems, which could have affected MS's manner of interviewing and interpreting. MS's personal and professional experience in substance addiction rehabilitation could have allowed him to discuss more elaborately and establish better rapport with the residents. Moreover, his personal connection to the issue compelled him to assume both clinician and researcher roles for a few residents who sought his professional advice during the interview. These instances where signposted in the audio recording with the utterance, "this is off the record [because it is not pertinent to the study]..." Table 3 outlines suggested competencies in administering the OJHQ-FV.

Table 3. List of interview competencies essential in using the OJHQ-FV within the substance addiction rehabilitation setting.

- Adjusting the line of questioning

- Ad-libbing (i.e., asking more or less questions outside of the script)

- Asking about sensitive and personal issues

- Changing topics

- Empathizing to respondents

- Interpreting stories and conversations

- Knowing about current and pressing issues in society

- Probing

- Prompt shifting of therapeutic use of self (or therapeutic communication) as needed

- Relating to different types of people with different backgrounds and experiences 
Included in the proposed guideline in OJHQ-FV administration are situational considerations which consist of unstructured observations of the physical space, sociocultural context, and gestures, as well as using the tool more than once. The facility informed the researchers that gesturing and using some forms of sign language were discouraged, rather words and verbalizations must be used to convey messages among the researchers and between researchers and residents to reinforce transparency; this is a rule applicable to all people visiting the facility. Accordingly, MS, CDR, and NR agreed that a specific socio-cultural influence governed the actions of all people who enter the facility. The reflective researchers also witnessed that the residents were expected to treat the researchers with respect. CDR surmised, "... [that] was one of the reasons why some [residents] initially acted distant, shy, and quiet." Because of the sensitive issue of substance addiction rehabilitation and the political stance of the Philippines on drug use, the reflective researchers proposed that the OJHQ-FV should be administered at least twice. Ideally, the first session is for administering the OJHQ-FV as an interview tool and to establish rapport with clients who were understandably cautious in sharing their injustice experiences. The second session is for verifying initial responses, completing unfinished interviews, and to capture a more developmental profiling of the clients in case there are changes in the clients' life situation (i.e., lawsuit verdict, family matters, and premature discharge among others).

\section{Theme 3: Contextualization of the OJHQ}

The third theme encapsulated the reflective researchers' expressions about how OJHQ-FV can be further contextualized where two subthemes emerged: item considerations (sub-theme 3.1) and suggestions on the process of further refining the OJHQ-FV (sub-theme 3.2).

Part of instrument development is recognizing item considerations and cultural adaptation issues such as related items, inapplicable items, and population-specific concerns. Related and repeating items were identified based on how the residents and reflective researchers attributed similar meanings to them (e.g., income [Part I], work = well-being [Part II], employment [Part III]; leisure = well-being [Part II], leisure [Part III]; rest [Part III], holidays [Part III]; education [Part I], education toward full development of personality [Part III]; community cultural life [Part III], cultural beliefs and customs [Part IV]). CDR wrote that "...asking the same items was distracting... and redundant items [...] need to be clustered". The reflective researchers also deemed some items inapplicable for the residents such as items in Part III (e.g., holidays, the arts, and scientific advancement) to which MS commented:

[...] We recognize that the OJHQ was created from a Western perspective and that some determinants in the OJHQ may be perceived differently by an individual from a developing country in Southeast Asia where arts and holidays are only applicable for the "rich" and not the general population.

Since the sample in the study were confined in a residential facility, some items (e.g., holidays and participation in government) were considered inapplicable since these could not be experienced while they were admitted and confined within a facility. However, 
since the OJHQ-FV required asking the experiences of the interviewees before and during rehabilitation, some of the aforementioned items were only given entries in the "before rehabilitation" comments section.

Based on the reflections collected, further suggestions in the refinement of the OJHQ$F V$ could include specifying target population, a more comprehensive definition for the items, resolving translation issues, and formatting and layout. In line with developing supporting materials, MS and NR noted, "The OJHQ format and administration must be adapted to different populations and settings like Filipinos recovering from drug addiction, Japanese elderly living alone, and so on". However, MS reiterated that making different versions of OJHQ for a certain population is not mandatory but "[...] orientation and training is needed before using the OJHQ in order to get the most out of the administration considering the [target] population". Although the reflective researchers agreed that a more comprehensive definition per item could have provided clarity and direction on how questions were asked and answered, MS defended, "The [confusing] issue was resolved when the OJHQ-FV users convened before and after collection periods to clarify terms and redo the questioning to the residents on the second session". Moreover, all reflective researchers agreed that some English items were not readily translatable to the Filipino language either because there is no direct translation, or the group of words may be interpreted in various ways. For instance, the term well-being had to be translated using four words in the Filipino language, that is, pagiging komportable, malusog, [at] masaya, which literally translates to being comfortable, healthy and happy. Simply put, some English constructs used in the OJHQ-FV had to be defined using several words or phrases to provide a suitable translation. The last refinement process is the instrument format and layout, which led the reflective researchers to generate a list of suggestions as outlined in Table 4.

\section{Theme 4: The nuances of occupational injustices in the substance addiction rehabilitation setting}

These reflection notes were collected after the study has been completed. The reflective researchers pondered on the residents' understanding of occupational justice and the influence of the residents' individual circumstances on their experience of occupational (in)justice.

According to MS, the residents had minimal to no idea of what occupational justice, and its components, was about-there was an ignorance of (in)justice. Given this, MS reflected based on his field notes that for the residents, "justice" meant "experiencing fairness", while "injustice" meant "experiencing suffering". Such understanding of (in)justice was contested by RCP's comment:

$[\ldots]$ is it really our responsibility to bring into their consciousness the injustices that they are unknowingly experiencing? I mean, I understand that knowing about the injustice is the first step to acting and working to change the situation. However, in the situation where change seems unlikely or excruciatingly difficult - for instance, solving poverty, corruption, et cetera - would it be better to keep them content and blind to the injustice? As the saying goes, 'ignorance is bliss'. 
Table 4. Suggestions on how to improve the format and layout of the OJHQ.

- Actions and Recommendations section must reflect outcome measures in occupational participation and occupational justice

- Adjust the script depending on the setting, population, or situation

- In Part $V$ (injustices checklist), add "Others: " because there could be other injustices not initially listed

- Items in the questionnaire should be phrased as questions

- Merging or clustering some related items

- The Comments Section (i.e., before and during rehabilitation partitions) should be adapted when used in another setting, population, or situation

- The OJHQ layout could be redesigned by a professional layout artist

- The reasons why a determinant was not achieved (i.e., Health, Political, Social, and Economic) should be deliberately used in providing context on the comments and narratives of the respondents

DGY supported RCP with his own reflections where he articulated that "[...] there are several things that might be considered an injustice from the assessors' eyes, but not in the eyes of those who are injusticed because injustice is just part of their reality".

In one of the virtual meetings, CDR and NR recalled that injustices were not eradicated or reduced via the drug rehabilitation program. Rather, these injustices "mutated" into some other forms of injustices that were socially constructed within the context of the drug rehabilitation center. MS recalled, "[...] one of the residents mentioned that as a gay person, he was not allowed to assume a leadership responsibility within the ward because the authorities presume that he would use his position to molest other male residents." CDR added, "[...] in the women's ward, they label those with homosexual orientation as 'home girls'... they were segregated in a certain area away from the other women to prevent eye contact and unwanted physical touch". Thus, from experiencing threat of violence and death outside the drug rehabilitation center, the cause of injustice has become homophobia with the health care workers becoming complicit to a system that perpetuates these sexist practices. The reflexive conversation went on by highlighting the concept of "double marginalization" among residents who also considered themselves as members of the lesbian, gay, bisexual, transgender, and queer (LGBTQ) community. Double marginalization connotes the further exclusion of an already marginalized individual or collective because of gender, ethnicity, age, religion, or other characteristics (Eneyew \& Mengistu, 2013). While ideally, the goal is to eradicate social inequalities and injustices, another critical point was raised by RCP:

Can it be that the drug rehabilitation center permits the perpetuation of injustice? From our previous discussion, some of the residents has become suki (patrons) of the center. That is to say, the residents have started to see the center as a haven where they can receive the basic needs they cannot otherwise have outside. Thus, the rehabilitation center's structure of focusing on the drug use problem without much consideration to the socioeconomic situation that is driving drug use in the first place has set-up this vicious cycle...

Given these critical discussions, the consideration of pluralistic viewpoints is necessary to either support or contest each other's opinions or reflections. In response, 
MS explained how the medicalization approach towards illicit drug use and addiction in the Philippines could have perpetuated the "revolving door phenomenon" which was described by RCP. Medicalization is a process wherein using illicit drugs presumes that all users are "addicted" to the drugs thus needing medical attention, even if it is not necessary. This notion of addiction-as-disease is problematic as it only looks at individualistic solutions rather than societal solutions and interventions that go beyond the four walls of the drug rehabilitation centers. From these examples, the authors deemed that the OJHQ-FV was not only able to recognize the injustices among persons and groups, but arguably able to raise consciousness about the interweaving and evolving nature of occupational injustices.

\section{Discussion}

This article described the authors' reflections in utilizing the OJHQ as a potential tool to enable justice work in the context of substance addiction rehabilitation setting in the Philippines. Through the use of fieldnotes analysis, the findings revealed four themes that encapsulated the experiences and reflections in administering the OJHQFV namely tool usefulness, administration guidelines, guidelines, contextualized tool use, and nuances of occupational injustices in the substance addiction rehabilitation setting. In this section, the authors also expressed their interpretations of the study findings based on their post-study reflections drawn from the iterative discussions done, online and offline, throughout the process of co-writing this article.

While the OJHQ has been made available (Wilcock \& Townsend, 2014, p. 548$549)$, it still has no clear guidelines for administration and documentation. Sames (2014) proposed two important considerations occupational therapists need to remember in OT documentation: 1) people form an impression of your professionalism and intelligence by reading what you write; 2) what you write can be used as evidence in a court proceeding, whether you are on trial or not. The former reinforces the need to further develop the OJHQ as an evidence-informed assessment tool, while the latter offers an opportunity for occupational therapists to advocate their roles not only within the health and social care settings but also in the legal and justice systems (Malfitano et al., 2019). Thus, engaging in translation (Whiteford \& Townsend, 2011; World Health Organization, 2020) and critical reflexive exercises (McCorquodale \& Kinsella, 2015) were strategies the authors employed to help attune the use of OJHQFV for future studies and practice in the Philippine context. The researchers were also cognizant that while employing these strategies, the processes of translation, adaptation, and experiencing technical issues are influential in cross-cultural adaptation of evaluation tools used in occupational therapy (Alotaibi, 2008).

The original purposes of the OJHQ are to "[...] prompt attention to occupational injustice in a busy practice day [...]" and to "[...] provide a starting point to document injustices and encourage action [...]" (Wilcock \& Townsend, 2014, p. 548). The first theme that emerged from this study attempted to expand the tool's purposes to 1) enabling narrative professional reasoning among practitioners and 2) extracting additional layers of non-clinical data. In the beginning of the OT process, an occupational therapist collaborates with a client to co-create their occupational profile "[...] which provides an understanding of the client's occupational [hi]story and 
experiences...” (American Occupational Therapy Association, 2020, p. 21). Using the OJHQ-FV allows the occurrence of emancipatory co-creation that produces life stories that not only identify injustice experiences, but also reinforce clients' ability to author their own intervention, discharge, and/or even self-advocacy plans. Such strategies, which engender client-centeredness and narrative clinical reasoning in practice, were considered as acts of justice within the scope of OT practice (Bailliard et al., 2020). Furthermore, utilizing the OJHQ to extract non-clinical data enables better understanding of available clinical data as it provides perspectives on the societal (structural and contextual) constructs that influence a person's health [condition] (Hammell, 2020). These non-clinical data sets could also aid occupational therapists who are just beginning to appreciate occupational justice in practice to create plans and recommendations according to the mico-, meso-, and macro levels of human functioning as proposed by Bailliard et al. (2020). Occupational injustices arise from complex transactional situations wherein innumerable and interdependent human and environmental factors meet in action (Kinsella \& Durocher, 2016). Because an occupational justice perspective entails the thorough and critical examination of societal constructs surrounding a person, the inclusion of these non-clinical data will challenge the oversimplified assessment underlying ineffective interventions (Bailliard, 2016) and thereby espouse assessments and interventions that go beyond the biomedical model of care (Yao et al., 2020).

The OJHQ was used in a recent single-subject case study and only provided brief guidelines in administering the OJHQ such as seeking permission and informed consent, face-to-face interview, and 90-minute interview time (Sy \& Ohshima, 2019). These three simple guidelines were used as the basis in formulating a more rigorous method of the study by Sy et al. (2019) where the OJHQ-FV was piloted and administered. To make these guidelines more relevant to the changing times, digital interviewing using the OJHQ as a tool could be possible with careful considerations on data privacy and confidentiality, internet connectivity, and providing alternatives such as mobile phones (Sy et al., 2020).

Derived from the reflection of the pilot test, the second and third themes offered suggestions in utilizing and improving the guidelines and overall instrument development of the OJHQ in the future. Proposing a 90-minute interview and allocating a separate session for verification, however, appeared to be counterintuitive to the first purpose of the OJHQ, which is "to prompt attention to occupational injustice in a busy practice day". This suggestion was made in consideration of the sensitive nature of substance addiction rehabilitation practice in the Philippines amidst the country's War on Drugs. Residents may be hesitant to share and state matters which may put them in a bad light. This phenomenon could be due to "social desirability bias", which is the tendency to underreport socially undesirable attitudes and behaviors and to over report more desirable attributes especially in the substance addiction rehabilitation setting (Latkin et al., 2017). In order to mitigate this bias, Latkin et al. (2017) suggested (re)wording and prefacing of questions, defining the role of the "study participant", and assessing and addressing motivations for socially desirable responses-all of which can be employed when using the OJHQ for future research and practice. Furthermore, the lack of a previously established rapport and power imbalance between residents and the reflective researchers of this study may elicit a more guarded response thus necessitating 
the need to verify information on a separate day. In addition to these suggestions, it should be highlighted that OJHQ users should be guided by an occupational justice framework to enact theory-based evaluation, intervention, and outcome measurement processes across different levels of human functioning.

It is equally important to further examine the usability of the OJHQ by not only blindly adapting the instrument but contextualizing its utility based on indigenous theories, participant voices, priorities, and cultural values (Jadhav, 2009), as well as introducing alternative and neutral terms that are non-binary and categorical (Twinley \& Castro de Jong, 2020). Human rights, the items enumerated within the OJHQ, are universal and equal to all people regardless of race, ethnicity, nationality, class, religion, gender, and language (United Nations Population Fund, 2005). Thus, there should be a common understanding through a comprehensive definition of each item (see Supplemental Document 2) to diminish issues and difficulties in interpreting and translating the OJHQ to other languages. However, despite the universality of the human rights concept, the authors argue that the implementation and manifestation of such rights needs to be reflective of personal, communal, and populational differences. This argument is supportive of Córdoba's (2020, p. 1365) reflection on occupational justice as a concept:

The scope of the idea of $O J$ and its colonial implications are delimited, an idea of Justice is proposed based on a critical ethical-political criterion, not an instrumental technical one, based on the idea of good living, under a pluricultural logic, of an anti-capitalist, communitarian order and that it materializes an expression of situated human rights, based on the recognition of difference.

Thus, this research, as reflected in the third theme, explored the cultural nuances of using the OJHQ in a non-Western and developing world setting. Despite human rights being operated under the notion of equality, several items were deemed inapplicable by either the reflective researchers or the facility residents due to the differences in interpreting the relativity of the concepts of justice and injustice. Apart from considering cultural sensitivity in instrument utilization, answering these questions can galvanize a more reflective, critical, and occupation-centered OT practice. Hence, we propose the importance of pilot testing the OJHQ before using in other populations, contexts, and settings to ensure the instrument's optimal utility.

The post-study reflections also revealed the interweaving and evolving nature of occupational injustices. In literature, occupational (in)justices are usually categorized to: deprivation, imbalance, alienation, marginalization, and apartheid (Kronenberg et al., 2005; Stadnyk et al., 2010; Hocking, 2017). However, ongoing critical reflections of the authors ascertained that in using the OJHQ-FV, occupational injustices cannot be boxed into categories since the injustices can be experienced and interpreted in multiple ways. Moreover, occupational therapists in substance addiction rehabilitation settings cannot just stop in the identification of injustices after occupational profiling, but must consider moving forward in the co-creation of solutions beyond individual care (Sy et al., 2019). Apart from partnering and collaborating with other health and social care professionals and organizations to promote occupational justice for people and groups, it is important to position justice-focused solutions from pluralistic perspectives on 
health, well-being, and justice (Bailliard, 2016) employed throughout the micro, meso-, and macro- levels of human functioning (Bailliard et al., 2020). Hence, it is crucial for occupational therapists and justice workers to be mindful about occupational justice terminologies when using the OJHQ in future practice and research. They should also be ready to engage in critical reflections as this will support the co-creation of collective and social professional actions as part of their occupational therapy practice (Malfitano et al., 2019).

This article recognizes that the data produced were reflective notes generated and analyzed by the first three authors of this paper in a very specific context. Consequently, the words used by the authors to convey their reflections are in English, their second language, which may be interpreted differently by various readers. Also, this study was methodologically limited because the last two authors were not able to use the OJHQ and only participated in the reflexive exercise towards the latter parts of the study. Due to this limitation, future studies using the OJHQ can include more OJHQ users, more respondents, and application in different contexts, cultures, and languages. We, therefore, caution readers that this study was done in one country and one setting and its implications may not be readily applicable across countries or settings. Nonetheless, the interview guide developed for the OJHQ-FV and the glossary of terms provided some structure for consistent definitions during tool administration without restricting its use in alternative practice settings.

\section{Conclusion}

Occupational justice remains a developing concept within OT practice. However, efforts to translate its theoretical underpinnings into tangible outcomes are now being advanced by occupational therapists, scientists, and instrument developers. This article describes a strategy for reflecting about the OJHQ to see its potentialities for adaptation in contexts where OT is practiced such as in substance addiction rehabilitation settings. Using a qualitative approach, specifically through the extraction of reflective field notes, findings revealed an expansion of OJHQ's use as an instrument such as enhancing practitioner's clinical and professional reasoning, facilitating justice-oriented practice across all levels of human functioning (individuals, groups, and populations), activating reflective and critical OT practice, and recognizing the interweaving and evolving nature of occupational injustices. In this era of rapid change and liminality, occupational therapists must deliberately engage in reflexive exercises in order to consider contexts with intentionality and thereby find concrete ways to adapt and respond to humans and their doings beyond health-related constructs during and even after the evaluation and intervention processes. By using the OJHQ alongside with other recognized tools within the scope of the OT profession, occupational therapists can potentially reimagine contextsensitive practices and espouse OT services underpinned by occupational justice.

\section{Acknowledgements}

The authors would like to express gratitude to Prof. Nobuo Ohshima, Dr. Adrian Paul Rabe, and Dr. Joaquin Antonio Benedicto for providing us with insightful comments during the conduct of the study. 


\section{References}

Alotaibi, N. M. (2008). The cross-cultural adaptation of the disability of arm, shoulder and hand (DASH): a systematic review. Occupational Therapy International, 15(3), 178-190. http://dx.doi.org/10.1002/oti.252.

American Occupational Therapy Association - AOTA. (2020). Occupational therapy practice framework: domain and process. North Bethesda: AOTA.

Bailliard, A. (2016). Justice, difference, and the capability to function. Journal of Occupational Science, 23(1), 3-16. http://dx.doi.org/10.1080/14427591.2014.957886.

Bailliard, A. L., Dallman, A. R., Carroll, A., Lee, B. D., \& Szendrey, S. (2020). Doing occupational justice: a central dimension of everyday occupational therapy practice. Canadian Journal of Occupational Therapy, 87(2), 144-152. http://dx.doi.org/10.1177/0008417419898930.

Bernardo, A. B. I. (2011). Lost In translation? Challenges in using psychological tests in the Philippines. Silliman Journal, 52(1), 21-45.

Bondoc, I. P., Mabag, V., Dacanay, C. A., \& Macapagal, N. D. (2017). Speech-language pathology research in the Philippines in retrospect: perspectives from a developing country. International Journal of Speech-Language Pathology, 19(6), 628-636. https://doi.org/10.1080/17549507.2016.1226954

Boyt Schell, B. A. (2014). Processional reasoning in practice. In B. A. Boyt Schell, G. Gillen, \& M. E. Scaffa (Eds.), Willard \& Spackman's occupational therapy (pp. 384-397). Philadelphia: Wolters Kluwer Health/Lippincott Williams \& Wilkins.

Collier-Reed, B. I., Ingerman, Å., \& Berglund, A. (2009). Reflections on trustworthiness in phenomenographic research: recognising purpose, context and change in the process of research. Education as Change, 13(2), 339-355. http://dx.doi.org/10.1080/16823200903234901.

Córdoba, A. G. (2020). About new forms of colonization in occupational therapy. Reflections on the Idea of Occupational Justice from a critical-political philosophy perspective. Cadernos Brasileiros de Terapia Ocupacional, 28(4), 1365-1381. http://dx.doi.org/10.4322/2526-8910.ctoarf2175.

Coronel, C. M. A. (2005). Towards occupation-based assessment for Filipino occupational therapists. Philippine. Journal of Occupational Therapy, 1(1), 30-36.

Creswell, J. W. (2014). Research design: qualitative, quantitative, and mixed methods approaches. California: SAGE Publications.

de Groot, A. M. (1992). Determinants of word translation. Journal of Experimental Psychology. Learning, Memory, and Cognition, 18(5), 1001-1018. http://dx.doi.org/10.1037/0278-7393.18.5.1001

Delos Reyes, R. C., Baes, D. M., Gualberto, S. M., \& Pagcaliwagan, E. (2021). Experiences of university students during COVID-19: A case study using occupational justice perspective. The Journal of School Health, 4(1), 1-9.

Durocher, E., Gibson, B., \& Rappolt, S. (2014). Occupational justice: a conceptual review. Journal of Occupational Science, 21(4), 418-430. http://dx.doi.org/10.1080/14427591.2013.775692.

Elo, S., \& Kyngäs, H. (2008). The qualitative content analysis process. Journal of Advanced Nursing, 62(1), 107-115. http://dx.doi.org/10.1111/j.1365-2648.2007.04569.x.

Eneyew, A., \& Mengistu, S. (2013). Double marginalized livelihoods: invisible gender inequality in pastoral societies. Societies, 3(1), 104-116. http://dx.doi.org/10.3390/soc3010104.

Enriquez, V. G. (1992). From colonial to liberation psychology. Quezon City: University of the Philippines Press.

Friese, S. (2013). ATLAS.ti 7 user manual. Berlin: ATLAS.ti.

Garcia, J. L. A. (2006). Sin and suffering in a Catholic understanding of medical ethics. Christian Bioethics, 12(2), 165-186. http://dx.doi.org/10.1080/13803600600805401.

Guanzon-Lapeña, M. A., Church, A. T., Carlota, A. J., \& Katigbak, M. S. (1998). Indigenous Personality Measures: philippine Examples. Journal of Cross-Cultural Psychology, 29(1), 249-270. https://doi.org/10.1177/0022022198291013 
Guba, E., \& Lincoln, Y. (1989). Fourth generation evaluation. Texas: SAGE Publications.

Gupta, J. (2016). Mapping the evolving ideas of occupational justice: a critical analysis. OTJR, 36(4), 179-194. http://dx.doi.org/10.1177/1539449216672171.

Hammell, K. (2017). Critical reflections on occupational justice: toward a rights-based approach to occupational opportunities. Canadian Journal of Occupational Therapy, 84(1), 47-57. http://dx.doi.org/10.1177/0008417416654501.

Hammell, K. W. (2020). Action on the social determinants of health: advancing occupational equity and occupational rights. Cadernos Brasileiros de Terapia Ocupacional, 28(1), 378-400. http://dx.doi.org/10.4322/2526-8910.ctoarf2052.

Hammell, K. W., \& Iwama, M. K. (2012). Well-being and occupational rights: an imperative for critical occupational therapy. Scandinavian Journal of Occupational Therapy, 19(5), 385-394. http://dx.doi.org/10.3109/11038128.2011.611821.

Hocking, C. (2017). Occupational justice as social justice: the moral claim for inclusion. Journal of Occupational Science, 24(1), 29-42. http://dx.doi.org/10.1080/14427591.2017.1294016.

Jadhav, D. (2009). What is cultural validity and why is it ignored? In S. V. D. Geest \& M. Tankink (Eds.), Theory and action: Essays for an anthropologist (pp. 92-96). Holanda: AMB Diemen.

Jasso, G. (2005). Culture and the sense of justice: A comprehensive framework for analysis. Journal of Cross-Cultural Psychology, 36(1), 14-47. http://dx.doi.org/10.1177/0022022104271425.

Jensen, S., \& Hapal, K. (2018). Police violence and corruption in the Philippines: violent exchange and the war on drugs. Journal of Current Southeast Asian Affairs, 37(2), 39-62. http://dx.doi.org/10.1177/186810341803700202.

Jensen, S., Hapal, K., \& Modvig, J. (2013). Violence in Bagong Silang: a research report prepared in collaboration between DIGNITY and Balay. Dinamarca: Danish Institute Against Torture.

Kennedy, J., \& Davis, J. A. (2017). Clarifying the construct of occupational engagement for occupational therapy practice. OTJR, 37(2), 98-108. http://dx.doi.org/10.1177/1539449216688201.

Kinsella, E. A., \& Durocher, E. (2016). Occupational justice: moral imagination, critical reflection, and political praxis. OTJR, 36(4), 163-166. http://dx.doi.org/10.1177/1539449216669458.

Kronenberg, F., Algado, S. S., \& Pollard, N. (2005). Occupational therapy without borders: learning through the spirit of survivors. Edinburgh: Elsevier/Churchill Livingstone.

Latkin, C. A., Edwards, C., Davey-Rothwell, M. A., \& Tobin, K. E. (2017). The relationship between social desirability bias and self-reports of health, substance use, and social network factors among urban substance users in Baltimore, Maryland. Addictive Behaviors, 73, 133-136. http://dx.doi.org/10.1016/j.addbeh.2017.05.005.

Leung, K., \& Stephan, W. G. (2001). Social justice from a cultural perspective. In D. Matsumoto (Ed.), Handbook of culture and psychology (pp. 375-410). Reino Unido: Oxford University Press.

Magno, C. (2010). A brief history of educational assessment in the Philippines. Educational Measurement and Evaluation Review, 1, 140-149.

Malfitano, A. P. S., de Souza, R. G. da M., Townsend, E. A., \& Lopes, R. E. (2019). Do occupational justice concepts inform occupational therapists' practice? A scoping review. Canadian Journal of Occupational Therapy, 86(4), 299-312. http://dx.doi.org/10.1177/0008417419833409.

Malfitano, A. P. S., de Souza, R. G. M., \& Lopes, R. E. (2016). Occupational justice and its related concepts: an historical and thematic scoping review. OTJR, 36(4), 167-178. http://dx.doi.org/10.1177/1539449216669133.

McCorquodale, L., \& Kinsella, E. A. (2015). Critical reflexivity in client-centred therapeutic relationships. Scandinavian Journal of Occupational Therapy, 22(4), 311-317. http://dx.doi.org/10.3109/11038128.2015.1018319.

Nilsson, I., \& Townsend, E. A. (2010). Occupational justice: bridging theory and practice. Scandinavian Journal of Occupational Therapy, 17(1), 57-63. http://dx.doi.org/10.3109/11038120903287182. 
Phillippi, J., \& Lauderdale, J. (2018). A guide to field notes for qualitative research: context and conversation. Qualitative Health Research, 28(3), 381-388. https://doi.org/10.1177\%2F1049732317697102.

Ryan, G. W., \& Bernard, H. R. (2003). Techniques to identify themes. Field Methods, 15(1), 85-109. http://dx.doi.org/10.1177/1525822X02239569.

Sames, K. M. (2014). Documentation in practice. In B. A. Boyt Schell, G. Gillen, \& M. E. Scaffa (Eds.), Willard \& Spackman's occupational therapy (pp. 467-475). Philadelphia: Lippincott Williams \& Wilkins.

See, S. E. (2008). Filipinos are punny. In P. Patajo-Legasto (Ed.), Philippine studies: have we gone beyond St. Louis? (pp. 683-713). Quezon: University of the Philippines Press.

Stadnyk, R., Townsend, E., \& Wilcock, A. (2010). Occupational justice. In C. H. Christiansen \& E. Townsend (Eds.), Introduction to occupation: The art and science of living (pp. 329-358). Londres: Pearson Education.

Sy, M. P., \& Ohshima, N. (2019). Utilizing the Occupational Justice Health Questionnaire (OJHQ) with a Filipino drug surrenderee in occupational therapy practice: a case report. World Federation of Occupational Therapists Bulletin, 75(1), 59-62. http://dx.doi.org/10.1080/14473828.2018.1505682.

Sy, M. P., Reyes, R., Roraldo, M., \& Ohshima, N. (2019). Uncovering the lived experiences of Filipino drug recoverees towards occupational participation and justice through an interpretative phenomenological analysis. Scandinavian Journal of Occupational Therapy, 1-14. http://dx.doi.org/10.1080/11038128.2019.1642380.

Sy, M., O’Leary, N., Nagraj, S., El-Awaisi, A., O’Carroll, V., \& Xyrichis, A. (2020). Doing interprofessional research in the COVID-19 era: a discussion paper. Journal of Interprofessional Care, 34(5), 600-606. http://dx.doi.org/10.1080/13561820.2020.1791808.

Twinley, R., \& Castro de Jong, D. (2020). Commentary on: Sy, Bontje, Ohshima \& Kiepek. Articulating the form, function, and meaning of drug using in the Philippines from the lens of morality and work ethics. Journal of Occupational Science, 27(1), 22-25. http://dx.doi.org/10.1080/14427591.2020.1733924.

United Nations Population Fund - UNFPA. (2005). Human rights principles. Boston: Harvard School of Public.

Whiteford, G., \& Townsend, E. (2011). Participatory Occupational Justice Framework (POJF 2010): Enabling occupational participation and inclusion. In F. Kronenberg, N. Pollard \& D. Sakellariou (Eds.), Occupational therapies without borders: Towards an ecology of occupation-based practices (pp. 65-84). Amsterdam: Elsevier.

Wilcock, A. A., \& Townsend, E. A. (2014). Occupational Justice Health Questionnaire. In B. A. Boyt Schell, G. Gillen \& M. E. Scaffa (Eds.), Willard \& Spackman's occupational therapy (pp. 548-549). Philadelphia: Lippincott Williams \& Wilkins.

World Health Organization - WHO. (2020). Management of substance abuse: process of translation and adaptation of instruments. Geneva: WHO.

Yao, D. P. G., Inoue, K., Sy, M. P., Bontje, P., Suyama, N., Yatsu, C., Perez, D. A., \& Ito, Y. (2020). Experience of Filipinos with spinal cord injury in the use of assistive technology: an occupational justice perspective. Occupational Therapy International, 2020(1), 1-10. http://dx.doi.org/10.1155/2020/6696296. 


\section{Author's Contributions}

Michael Palapal Sy and Rod Charlie Delos Reyes were responsible for conceptualization, literature review, data collection and analyses, and writing of the manuscript. Ma. Patricia Nicole Ragunton Roraldo was responsible for conceptualization, data collection and analyses and writing of the manuscript. Daryl Patrick Gamboa Yao was responsible for literature review and writing of the manuscript. Roi Charles Sarmiento Pineda was responsible for writing of the manuscript. All authors approved the final version of the text.

\section{Funding Source}

This work was supported by Tokyo Metropolitan University Urban Diplomatic Personnel Grant Fund.

\section{Corresponding author}

Michael Palapal Sy

E-mail: mpsy@up.edu.ph

\section{Section editor}

Profa. Dra. Ana Paula Serrata Malfitano 


\section{Supplementary Material}

Supplementary material accompanies this paper.

Supplemental Document 1. Occupational Justice Health Questionnaire (Filipino Version).

Supplemental Document 2. Definition of Constructs in the Occupational Justice Heath Questionnaire.

This material is available as part of the online article from http://www.scielo.br/CTO 